Bull. Austral. Math. Soc.

VoL. 44 (1991) [357-365]

\title{
THE CENTRALISER OF THE INJECTIVE TENSOR PRODUCT WEND WERNER
}

The aim of this note is to obtain an intrinsic product formula for the centraliser of the injective tensor product of a couple of Banach spaces, $Z\left(X \widehat{\otimes}_{\varepsilon} Y\right)$. More precisely, we are going to prove that

$$
Z\left(X \widehat{\otimes}_{c} Y\right)=C^{b}\left(Z_{X} / \mathfrak{F}_{X} \times_{k} Z_{Y} / \mathfrak{F}_{Y}\right)
$$

Here, the spaces $Z_{X} / \mathfrak{F}_{X}$ and $Z_{Y} / F_{Y}$ depend only on $X$ and $Y$, respectively, and $x_{k}$ denotes the topological k-product.

A counterexample used to demonstrate that the $k$-product cannot be avoided serves also as an answer to a question posed by $W$. Rue $B$ and $D$. Werner concerning the behaviour of $M$-ideals on $X \widehat{\otimes}_{\varepsilon} Y$.

\section{INTRODUCTION}

Let $X$ be a Banach space, $B_{X}$ its unit ball and denote by ex $K$ the set of extreme points of some subset $K \subseteq X$. Suppose for the moment that $X$ is a real space and put

$$
Z(X):=\left\{T \in L(X) \mid \forall p \in \operatorname{ex} B_{X^{\prime}} \exists a_{T}(p) \in \mathbb{R} \quad T^{\prime} p=a_{T}(p) p\right\}
$$

In the operator norm, $Z(X)$ is a commutative $C^{*}$-algebra. (For the definition in the complex case see the following section.)

The aim of the present note is to obtain an intrinsic product formula for $Z\left(X \widehat{\otimes}_{\varepsilon} Y\right)$, that is, an expression which does not resort to any properties of the injective tensor product as such. More precisely, we are going to show that the equation

$$
Z\left(X \widehat{\otimes}_{\varepsilon} Y\right)=C^{b}\left(Z_{X} / \mathfrak{F}_{X} \times_{k} Z_{Y} / \mathfrak{F}_{Y}\right)
$$

holds within the frame of Banach algebras. Here, the spaces $Z_{X} / \mathfrak{F}_{X}$ and $Z_{Y} / \mathfrak{F}_{Y}$ depend only on $X$ and $Y$, respectively, and $x_{k}$ denotes the topological k-product.

A related formula was obtained in [24], where it was shown that

$$
Z\left(X \widehat{\otimes}_{e} Y\right)=[Z(X) \otimes Z(Y)]^{-}
$$

Received 14 November 1990

Copyright Clearance Centre, Inc. Serial-fee code: 0004-9729/91 \$A2.00+0.00. 
Here, the closure has to be taken with respect to the strong operator topology on the space $X \widehat{\otimes}_{\varepsilon} Y$. For a different approach to this result see [3]. (For some similar results in more special situations the reader is referred to $[3,5,13,14,22,23]$ where, however, sometimes a slightly different notation is used.)

Let us indicate the source of interest in $Z(X)$. First, its subalgebras appear quite naturally whenever $X$ is represented as a space of (all continuous) sections in a Banach bundle, and in fact the whole algebra gives rise to such a representation which in some sense is maximal. (See [4]; in light of this property of $Z(X)$, the above equation can be used to obtain a maximal bundle representation of the injective tensor product without any of the restrictions on the involved Banach spaces as in [3] - but we won't touch this here.) The interest in Banach bundles in turn is manifold, see for example [4, 9, 11]. In [6] this concept has recently become a tool in the biholomorphic classification of domains in infinite dimensional Banach spaces. (Note, however, that the pertinent definitions frequently differ.) Second, in the theory of non associative Banach algebras, $Z(A)$ quite often provides a concept of centroid, which seems to be more manageable than the pure algebraic definition. For a more recent application of this sort see [17]. For the question of how $Z(X)$ looks like in some more concrete examples, the reader is referred to the following section.

Let us explain how this paper is organised: The following section collects some necessary notation as well as two auxiliary results. To one of them, a theorem of Stone-Weierstraß type, we briefly sketch some further applications. In the third section we state and prove our main theorem. We finally present an example in Section 4 that serves for two purposes: First, it provides a counterexample to a more ambitious conjecture in connection with the main result. On the other hand, it answers a question of W. RueB and D. Werner posed in [20].

\section{NOtATION AND USEFUL RESUltS}

We begin with

Definition 1: The Banach algebra Mult $X$ consists of all those operators $T$ for which each $p \in \operatorname{ex} B_{X^{\prime}}$ is an eigenvector of $T^{\prime}$ with eigenvalue $a_{T}(p)$.

Those $T \in M u l t X$ that possess a natural adjoint in Mult $X$, that is for which there exists $T^{*} \in M u l t X$ with $a_{T^{*}}(p)=\overline{a_{T}(p)}$ for all $p \in \operatorname{ex} B_{X^{\prime}}$, are said to belong to the centraliser, denoted by $Z(X)$.

Clearly, when $X$ is a real space, both algebras coincide. Note that both algebras are function algebras and that $Z(X)$ is a $C K$-space for a suitable compact $K$. For a more detailed presentation of this topic see [4].

Suppose that $X$ is a closed subspace of $C_{0} L$, the space of all continuous functions 
on the locally compact space $L$ vanishing at infinity, and let

$$
\begin{aligned}
\operatorname{Mult}\left(X, C_{0} L\right) & :=\left\{f \in C^{b} L \mid f X \subseteq X\right\} \\
Z\left(X, C_{0} L\right) & :=\left\{f \in M u l t\left(X, C_{0} L\right) \mid \bar{f} \in M u l t\left(X, C_{0} L\right)\right\}
\end{aligned}
$$

We further denote by

$$
\mathfrak{F}\left(X, C_{0} L\right)
$$

the set of equivalence classes which are obtained by

$$
l \sim k \Longleftrightarrow f(l)=f(k) \quad \forall f \in Z\left(X, C_{0} L\right) .
$$

The reader should observe that $Z\left(X, C_{0} L\right)$ is always a closed subalgebra of $Z(X)$. Furthermore, when $X$ is canonically embedded into the space $C_{0} Z_{X}$, where $Z_{X}:=$ $\overline{\operatorname{ex} B_{X^{\prime}}} \omega^{*} \backslash\{0\}$, then $Z(X)=Z\left(X, C_{0} Z_{X}\right)$ as well as $\mathfrak{F}\left(X, C_{0} Z_{X}\right)=\mathfrak{F}_{X}$. A result similar to the following can be found in [10, Theorem 13.2].

THEOREM 2. Let $X$ be a closed subspace of $C_{0} L$. Then $f \in C_{0} L$ belongs to $X$ if and only if

$$
f_{\mid F} \in X_{\mid F} \quad \forall F \in \mathfrak{F}\left(X, C_{0} L\right) .
$$

The proof of this theorem is nothing but a slight modification of the argument Glicksberg gave in order to prove Bishop's version of the classical Stone-Weierstra $B$ theorem (see for example [10]), and in fact, if $X$ is a function algebra then Theorem 2 reduces to Bishop's theorem. (Note that in this case $\mathfrak{F}_{X}$ is the maximal antisymmetric decomposition of $X$ 's Shilov boundary.) We therefore omit it. Instead, let us see what is going on for special spaces:

\section{Corollary 3.}

(i) $A C^{*}$-algebra $A$ is commutative if and only if its centroid separates the points in the $w^{*}$-closure of the set of pure states of $A$.

(ii) A compact convex set $K$ in a LCTVS is a Bauer simplex if and only if the order bounded operators on $A(K)$ separate the points in $\overline{\operatorname{ex} K}$.

(iii) Denote by $\left(Z_{X}\right)_{\sigma}$ the quotient space obtained from identifying points of the form $\gamma p$ with $|\gamma|=1$. Then $X$ is a $C_{\sigma}$-space if and only if $Z(X)$ separates the points of $\left(Z_{X}\right)_{\sigma}$.

Let us briefly sketch the proofs : For (i), one has to use the fact that for $C^{*}$-algebras $Z(A)$ coincides with the centroid of $A,[12]$. In the unital case, this is of course a special case of Théoreme 11.3.1 in [7]. To see why (ii) holds, one has to take into account that an operator $T$ on $A(K)$ is order bounded if and only if $T \in Z(A(K))$, see [1, II Section 7], and that the Bauer simplices represent precisely the sets $M_{1}^{+}(C)$ for some 
compact space $C$ [1, II Section 4]. The statement of (iii), the proof of which follows readily from Theorem 2 and $[15$, p.218], should be compared to the central results of [18] and [21], where two other classes of $L^{1}$-preduals are classified in a similar way. To see this connection (and for the sake of preparing the counterexample announced in the introduction), we need

THEOREM 4. ([2]) Denote by (ex $\left.B_{X^{\prime}}\right)_{\sigma}$ the space obtained from ex $B_{X^{\prime}}$ by identifying points of the form $\gamma p$ with $|\gamma|=1, p \in \operatorname{ex} B_{X^{\prime}}$. Then the sets of the form

$$
\left(\operatorname{ex} B_{X^{\prime}} \cap J^{\circ}\right)_{\sigma}
$$

where $J$ runs through the $M$-ideals of $X$, form the closed sets of a topology called the structure topology of $X$.

Recall that a subspace $J$ of a Banach space $X$ is called an $M$-ideal, if and only if for some subspace $J^{*}$ of $X^{\prime}$

$$
X^{\prime}=J^{\circ} \oplus_{1} J^{*} .
$$

The point here is that the functions $a_{T}$ (introduced in Definition 1) correspond to the bounded structurally continuous functions (see [4, Chapter 3]). Now, in [18] the $L^{1}$ preduals with the property that the elements of $Z(X)$ separate the points of (ex $\left.B_{X^{\prime}}\right)_{\sigma}$ have been characterised, whereas in [21] it was shown that a Banach space is $G$-space if and only if (ex $\left.B_{X^{\prime}}\right)_{\sigma}$ is Hausdorff.

Let us finally point out that the version of the Stone-WeierstraB theorem which is valid in the context of function modules on some compact space $K$ (see for example [11]), can also be obtained using Theorem 2 .

The reason we are interested in Theorem 2 at this place is

Corollary 5. Denote by $\mathfrak{F}_{X}$ the set of equivalence classes on $Z_{X}$ defined by

$$
p \sim q \Longleftrightarrow \Phi^{\prime} p=\Phi^{\prime} q \quad \forall \Phi \in Z(X) .
$$

The algebra $Z\left(X, C_{0} L\right)$ consists exactly of those $f \in C^{b} L$ which are constant on each $F \in \mathfrak{F}_{X}$.

We have to fix some further notation: Let $T$ be a Hausdorff space. The space $k(T)$ is the set $T$ together with the topology in which a set is open if and only if its intersection with the compact subsets of $T$ is (relatively) open. $k(T)$ belongs to the class of $k$-spaces, which means that its topology is generated by the compact subsets of $k(T)$. In the same vein, the mapping

$$
k(f): k\left(T_{1}\right) \rightarrow k\left(T_{2}\right)
$$


differs from $f: T_{1} \rightarrow T_{2}$ by change of topologies only, and it is continuous whenever $f$ is. We will also follow the convention to write

$$
T_{1} \times_{k} T_{2}:=k\left(T_{1} \times T_{2}\right)
$$

The most exhaustive reference on this topic known to the author is [8]. The following lemma contains the topological ingredients of the proof of Theorem 7. Since we couldn't locate one in the literature, we include a proof.

LEMMA 6. Suppose that $T_{1,2}$ are Hausdorff spaces, that $T_{1}$ is locally compact, and that there are given equivalence relations $R_{1,2}$ on $T_{1,2}$ with appertaining quotient maps $\pi_{1,2}$ such that $T_{i} / R_{i}$ is Hausdorff and the space $\left(T_{1} \times T_{2}\right) /\left(R_{1} \times R_{2}\right)$ is a k-space. Then

$$
\left(T_{1} \times T_{2}\right) /\left(R_{1} \times R_{2}\right) \cong T_{1} / R_{1} \times{ }_{k} T_{2} / R_{2},
$$

where the homeomorphism is given by $k(H)$ with

$$
H\left(\left[\left(t_{1}, t_{2}\right)\right]\right)=\left(\left[t_{1}\right],\left[t_{2}\right]\right)
$$

Here, $[\cdots]$ refers to the formation of equivalence classes in either of the equivalence relations.

ProOF: By definition of the respective topologies, $H$ and hence $k(H)$ are continuous. Thus we are left with showing that the map $k(H)^{-1}=k\left(H^{-1}\right)$ is continuous, which is the same as showing that $H^{-1}$ is continuous when restricted to compact subsets $K$ of $T_{1} / R_{1} \times T_{2} / R_{2}$. By assumption on $T_{i} / R_{i}$, we may think of $K$ as having the form $K=K_{1} \times K_{2}$ with $K_{i}$ compact in $T_{i} / R_{i}$. Denote by $\pi_{12}$ the quotient mapping that belongs to the relation $R_{1} \times R_{2}$ on $T_{1} \times T_{2}$. By $[8,3.3 .28]$,

$$
\psi:=\pi_{1} \times\left.\pi_{2}\right|_{\pi_{1}^{-1}\left(K_{1}\right) \times \pi_{2}^{-1}\left(K_{2}\right)}
$$

is a quotient map and so the continuity of $H^{-1}{ }_{\mid K}$ follows from the fact that

$$
H^{-1}\left|K \psi=\pi_{12}\right|_{\pi_{1}^{-1}\left(K_{1}\right) \times \pi_{2}^{-1}\left(\kappa_{2}\right)}
$$

is continuous.

\section{MAIN THEOREM AND PROOF}

Theorem 7. For Banach spaces $X$ and $Y$ we have

$$
Z\left(X \widehat{\otimes}_{\varepsilon} Y\right) \cong C^{b}\left(Z_{X} / \mathfrak{F}_{X} \times_{k} Z_{Y} / \mathfrak{F}_{Y}\right)
$$


where the (algebraic) isomorphism between these spaces can be chosen so that the operator $\sum T_{i} \otimes S_{i}$, which is in $Z\left(X \widehat{\otimes}_{e} Y\right)$, may be identified with the map $\sum a_{T_{i}} \otimes a_{S_{i}}$.

Note that the quotient spaces involved need not be completely regular. Therefore the Gelfand space of $Z\left(X \widehat{\otimes}_{e} Y\right)$ has to be written

$$
\beta \varrho\left(Z_{X} / \mathfrak{F}_{X} \times_{k} Z_{Y} / \mathfrak{F}_{Y}\right)
$$

where $e T$ denotes the complete regularisation of $T$, which in our case is nothing but the weak $C^{b} T$ topology of $T$.

ProoF: In the following we shall make use of the fact that $Z_{X \widehat{\otimes}_{c} Y}=Z_{X} \otimes Z_{Y}$, which follows from results in [19] and [16]. Our proof consists mainly in showing that

$$
\mathfrak{F}_{X \widehat{\otimes}_{\mathbf{C}} Y}=\mathfrak{F}_{X} \otimes \mathfrak{F}_{Y}
$$

To show this, observe first that for $p \in Z_{X}$ and $f \in X \widehat{\otimes}_{c} Y$

$$
f_{p}(t):=f(p \otimes t) \quad t \in Z_{Y}
$$

belongs to $Y$. Analogously, $f^{q}$ belongs to $X$ for each $q \in Z_{Y}$. Representing $Z\left(X \widehat{\otimes}_{e} Y\right)$ as a space of bounded continuous functions on $Z_{X \widehat{\otimes}_{c} Y}$ we may define $\Phi_{p}$ with $p \in Z_{X}$ as above. We have for $e \in X$ with $p(e)=1$

$$
\Phi_{p} x=\Phi(p, \cdot) p(e) y(\cdot)=[\Phi(e \otimes y)]_{p}
$$

and so, by the Bishop-Phelps Theorem, $\Phi_{p} \in M u l t Y$. Since $\overline{\Phi_{p}}=(\Phi)_{p}$ we also have $\Phi_{p} \in Z(Y)$. In the same way, $\Phi^{q} \in Z(X)$ for all $q \in Y$. Now let $\xi_{1,2} \otimes \eta_{1,2} \in F \otimes G \in$ $\mathfrak{F}_{X} \otimes \mathfrak{F}_{Y}$. Then

$$
\Phi\left(\xi_{1} \otimes \eta_{1}\right)=\Phi_{\xi_{1}}\left(\eta_{1}\right)=\Phi_{\xi_{1}}\left(\eta_{2}\right)=\Phi_{\eta_{2}}\left(\xi_{1}\right)=\Phi_{\eta_{2}}\left(\xi_{2}\right)=\Phi\left(\xi_{2} \otimes \eta_{2}\right)
$$

and thus, each $\Phi \in Z\left(X \widehat{\otimes}_{e} Y\right)$ is constant on $F \otimes G$. On the other hand, by definition of $\mathfrak{F}_{X}$ and $\mathfrak{F}_{Y}$, two different sets $F_{1} \otimes G_{1}$ and $F_{2} \otimes G_{2}$ in $\mathfrak{F}_{X} \otimes \mathfrak{F}_{Y}$ are separated by elements $\Xi \otimes \Psi \in Z(X) \otimes Z(Y) \subseteq Z\left(X \hat{\otimes}_{c} Y\right)$, which settles our claim.

To finish the proof, let $\tau$ and $\pi_{X}$ denote the quotient maps from $Z_{X} \times Z_{Y}$ to $Z_{X} \otimes Z_{Y}$ and from $Z_{X}$ to $Z_{X} / \mathfrak{F}_{X}$, respectively. Clearly, the quotient topologies on $Z_{X \widehat{\otimes}_{\epsilon} Y} / \mathfrak{F}_{X \widehat{\otimes}_{\epsilon} Y}$ induced by $\pi_{X \widehat{\otimes}_{\varepsilon} Y}$ and $\pi_{X \widehat{\otimes}_{\varepsilon} Y} \circ \tau$ coincide, and because

$$
\pi_{X \widehat{\otimes}_{\mathbf{C}} Y} \circ \tau=\pi_{X} \times \pi_{Y}
$$

we may use Lemma 6 to obtain (note that the class of $k$-spaces is stable under the formation of quotient mappings)

$$
Z_{X \widehat{\otimes}_{\varepsilon} Y} / \mathfrak{F}_{X \widehat{\otimes}_{\mathfrak{\varepsilon}}} \cong\left(Z_{X} \times Z_{Y}\right) /\left(\mathfrak{F}_{X} \times \mathfrak{F}_{Y}\right) \cong Z_{X} / \mathfrak{F}_{X} \times_{k} Z_{Y} / \mathfrak{F}_{Y}
$$

By Corollary 5 we are done.

The following corollary is essentially known (combine Example 5 on page 155 of [4] with Theorem 4.5 of [3]). 
Corollary 8. Suppose that $X$ and $Y$ are dual spaces. Then

$$
Z\left(X \widehat{\otimes}_{e} Y\right)=Z(X) \widehat{\otimes}_{e} Z(Y)
$$

ProOF: To keep this proof within reasonable limits, we adopt the notation of [4, Chapter 4]. It is not very difficult to see that a maximal function module representation of a Banach space $X$ can be obtained by putting $K_{X}^{*}:=Z_{X} / \mathfrak{F}_{X}, K_{X}:=\beta K_{X}^{*}$, choosing the fibre above $F \in \mathfrak{F}_{X}$ to be $X_{\mid F}$ (this is in fact a Banach space) and to be $\{0\}$ elsewhere, and, finally, letting $\|x(F)\|=\left\|x_{\mid F}\right\|$. Theorem 5.13 of [4] then shows that in each dual space $X$ there is an element $e \in X$ such that

$$
\left\|e_{\mid F}\right\|=1 \quad \forall F \in \mathfrak{F}_{X}
$$

But $\left\{F \in Z_{X} / F_{X} \mid\left\|x_{\mid F}\right\| \geqslant \alpha\right\}$ is compact for all $x \in X$ and for each $\alpha>0$ and hence, $Z_{X} / \mathfrak{F}_{X}$ is compact. The conclusion follows from this.

Observe that in the above proof we have essentially profited from the compactness of the space $Z_{X} / \mathfrak{F}_{\boldsymbol{X}}$. With a similar reasoning, the above proof transfers to the case of $A(K)$-spaces and unital $C^{*}$-algebras.

\section{AN example}

Let us first observe that in general the statement of Corollary 4.2 is wrong: Whenever $L_{1,2}$ are locally compact spaces, then

whereas

$$
Z\left(C_{0} L_{1} \widehat{\otimes}_{e} C_{0} L_{2}\right) \cong C \beta\left(L_{1} \times L_{2}\right)
$$

$$
Z\left(C_{0} L_{1}\right) \widehat{\otimes}_{\varepsilon} Z\left(C_{0} L_{2}\right) \cong C\left(\beta L_{1} \times \beta L_{2}\right) \text {. }
$$

However, these two spaces are known to be different in general $[8,3.12 .21]$.

The following example shows that one cannot dispose of the index $k$ in the statement of Theorem 7: Let $X=\left\{f \in C_{0} \mathbb{R} \mid n f(n)=f(1) \forall n \in \mathbb{N}\right\}$. We have $Z_{X}=\left\{ \pm \delta_{k} \mid k \in \mathbb{R}\right\}$ and so $Z(X)=\left\{f \in C^{b} \mathbb{R} \mid f_{\mid \mathbb{N}}=\right.$ constant $\}$. It is also straightforward to check that $X \widehat{\otimes}_{\varepsilon} X=\left\{f \in C_{0} \mathbb{R}^{2} \mid m n f(m, n)=f(1,1)\right\}$ as well as $Z\left(X \hat{\otimes}_{\varepsilon} X\right)=C^{b} \mathbb{R}^{2} / \mathbb{N}^{2}$. We will show that $C^{b} \mathbb{R}^{2} / \mathbb{N}^{2} \neq C^{b}(\mathbb{R} / \mathbb{N})^{2}$. To this end, denote for $m, n \in \mathbb{N}$ by $D_{m, n}$ the (open) disk with radius $(m+n)^{-1}$ centered at $(m, n)$. Let $f$ be any function $f \in C^{b} \mathbb{R}^{2}$ that vanishes on $\mathbb{R}^{2} \backslash \bigcup_{m, n=1}^{\infty} D_{m, n}$ and attains the value 1 on $\mathbb{N}^{2}$. Since a neighbourhood of $\mathbb{N}$ always contains a set of the form $\left.\sum_{\mu \in \mathbb{N}}\right] a_{\mu}, b_{\mu}[$ with $\mu \in] a_{\mu}, b_{\mu}\left[, f\right.$ cannot be continuous when it is considered as a function on $(\mathbb{R} / \mathbb{N})^{2}$.

Pursuing the above example a little further, we arrive at 
Proposition 9. In general, the structure topology on $\left(\text { ex } B_{X \widehat{\otimes}_{e} Y}\right)_{\sigma}$ is not the product of the structure topologies of $\left(\operatorname{ex} B_{X}\right)_{\sigma}$ and $\left(\operatorname{ex} B_{Y}\right)_{\sigma}$.

This gives an answer to a question posed in [20]. Note that, as an equation of sets, we always have

$$
\left(\operatorname{ex} B_{X \widehat{\otimes}_{c} Y}\right)_{\sigma}=\left(\operatorname{ex} B_{X}\right)_{\sigma} \times\left(\mathrm{ex} B_{Y}\right)_{\sigma} .
$$

Proof: In fact, since the space $X$ constructed above is a $G$-space, one can use [21, Theorem 97] and the fact that

$$
\text { ex } B_{X^{\prime}}=\left\{ \pm \delta_{k} \mid k \in \mathbb{R} \backslash\{2,3, \ldots\}\right\}
$$

to see that (ex $\left.B_{X^{\prime}}\right)_{\sigma}$ provided with the structure topology is homeomorphic to $\mathbb{R} / \mathbb{N}$. But then

$$
\left(\operatorname{ex} B_{X^{\prime}}\right)_{\sigma} \times\left(\operatorname{ex} B_{X^{\prime}}\right)_{\sigma} \not\left(\operatorname{ex~} B\left(X \widehat{\theta}_{\varepsilon} X\right)^{\prime}\right)_{\sigma},
$$

since the latter space provided with the structure topology is homeomorphic with $\mathbb{R}^{2} / \mathbb{N}^{2}$.

\section{REFERENCES}

[1] E. M. Alfsen, Compact convex sets and boundary integrals (Springer-Verlag, Berlin, Heidelberg, New York, 1971).

[2] E. M. Alfsen and E. G. Effros, 'Structure in real Banach spaces. Part I and II', Ann. of Math. 96 (1972), 98-173.

[3] E. Behrends, 'The centralizer of tensor products of Banach spaces (a function space representation)', Pacific J. Math. 81 (1979), 291-301.

[4] E. Behrends, $M$-Structure and the Banach-Stone Theorem: Lecture Notes in Math. 736 (Springer-Verlag, Berlin, Heidelberg, New York, 1979).

[5] C.-H. Chu, 'On standard elements and tensor products of compact convex sets', J. London Math. Soc. 14 (1976), 71-78.

[6] S. Dineen, M. Klimek, and R.M. Timoney, 'Biholomorphic mappings and Banach function modules', J. Reine Angew. Math. 387 (1988), 122-147.

[7] J. Dixmier, Les $C^{*}$-algèbres et leurs représentations, second edition (Gauthier-Villars, Paris, 1969).

[8] R. Engelking, General topology (Warszawa, 1977).

[9] J.M.G. Fell and R.S. Doran, Representations of *algebras, locally compact groups, and *-alebraic bundles I,II: Pure Appl. Math 125, 126 (Academic Press, New York, London, 1988).

[10] T. W. Gamelin, Uniform algebras (Prentice-Hall, Englewood Cliffs, 1969).

[11] G. Gierz, Bundles of topological vector spaces and their duality: Lecture Notes in Math. 955 (Springer-Verlag, Berlin, Heidelberg, New York, 1982). 
[12] P. Harmand, D. Werner, and W. Werner, ' $M$-ideals in Banach spaces and Banach algebras', (in preparation).

[13] R. Haydon and S. Wassermann., 'A commutation result for tensor products of $C^{*}$-algebras', Bull. London Math. Soc. 5 (1973), 283-287.

[14] J.W. Kitchen and D.A. Robbins, 'Tensor products of Banach bundles', Pacific J. Math. 94 (1981), 151-16.

[15] H.E. Lacey, The isometric theory of classical Banach spaces (Springer-Verlag, Berlin, Heidelberg, New York, 1974).

[16] A. Lima and G. Olsen, 'Extreme points in duals of complex operator spaces', Proc. Amer. Math. Soc. 84 (1985), 437-440.

[17] A. Rodríguez-Palacios and A.R. Villena Muñoz, 'Centroid and extended centroid of $J B^{*}$-algebras', (preprint 1989).

[18] N.M. Roy, 'A characterization of square Banach spaces', Israel J. Math. 17 (1974), 142-148.

[19] W. Ruess and Ch. Stegall, 'Extreme points in duals of operator spaces', Math. Ann. 261 (1982), 535-546.

[20] W. Ruess and D. Werner, 'Structural properties of operator spaces', Acta Univ. Carolin. - Math. Phys. 28 (1987), 127-136.

[21] U. Uttersrud, 'On $M$-ideals and the Alfsen-Effros structure topology', Math. Scand. 43 (1978), 369-381.

[22] G. F. Vincent-Smith, 'The centre of the tensor product of $A(K)$-spaces and $C^{*}$-algebras', Quart. J. Math. Oxford Ser. 228 (1977), 87-91.

[23] W. Werner, 'Some results concerning the $M$-structure of operator spaces', Math. Ann. 282 (1988), 545-553.

[24] A. W. Wickstead, 'The centraliser of $E \otimes_{\lambda} F^{\prime}$, Pacific J. Math. 65 (1976), 563-571.

\author{
Department of Mathematics \\ Universitāt - Gesamthochschule Paderborn \\ Fachbereich 17 Postfach 1621 \\ Paderborn \\ Federal Republic of Germany
}

